\title{
Light sterile neutrino oscillation searches
}

\section{Sergeyeva Viktoriya*t}

Laboratoire d'Annecy de Physique des Particules, IN2P3, CNRS France

E-mail: viktoriya_srgyv@hotmail.com

Sterile neutrino mass scale is a free parameter. Thus, $v_{\text {sterile }}$ might exist within the energy range from $\sim \mathrm{eV}$ to $10^{15} \mathrm{GeV}$. Heavy $v_{\text {sterile }}$, with energies between $\sim \mathrm{keV}$ and $10^{15} \mathrm{GeV}$, are motivated by Grand Unified Theory and might explain Universe evolution and baryon asymmetry, they will not be discussed here.

This review is focused on light $v_{\text {sterile }}$ which energy is $\sim \mathrm{eV}$. Light sterile neutrino searches were motivated by anomalies such as LSND, Gallium and reactor antineutrino anomaly. Summary of anomalies is presented here. Major part of the review is dedicated to experimental results obtained with the recent very short baseline reactor experiments such as ratios of spectra at different baselines, exclusion plots. General conlusion and future prospects end this review.

Neutrino Oscillation Workshop (NOW2018)

9 - 16 September, 2018

Rosa Marina (Ostuni, Brindisi, Italy)

\footnotetext{
*Speaker.

${ }^{\dagger}$ Member of the STEREO collaboration.
} 


\section{Anomalies observation: hints of $v_{\text {sterile }}$ or experimental artefact?}

The first differences between experimental results and theory predictions of neutrino fluxes were observed in both reactor and accelerator experiments. The most mentioned ones are Gallex [1, 2], Reactor Antineutrino [3] and LSND [4] anomalies.

Gallium experiments showed a difference between measured and expected ${ }^{71} \mathrm{Ga}$ rates from detector calibration campaigns using intense ${ }^{51} \mathrm{Cr}$ and ${ }^{37} \mathrm{Ar}$ radioactive sources. Anomaly statistically significant at a level of about $\sim 3.0 \sigma$ still has no clear explanation today and motivates light $v_{\text {sterile }}$ searches.

Reactor antineutrino experiments showed a difference between measured and expected neutrino flux from the reactor. Today, the mean ratio of $N_{o b s} / N_{\text {pred }}=0.943 \pm 0.023<1$, represents the $v_{e}$ disappearance anomaly, also called as Reactor Antineutrino Anomaly (RAA). Several possible explanations were suggested. The first one considers that there is an erroneous prediciton of reactor neutrino flux. The second one considers the possible existence of light $v_{\text {sterile }}$ and suggests the $3+1$ neutrino framework. Both ways are being actively studied nowadays by the neutrino community. For this review, we will stay in the frame of $3+1$ neutrino hypothesis.

The first combination of Gallium and reactor anomalies provided allowed regions for $\Delta m_{n e w}^{2}$ and $\sin ^{2}\left(2 \Theta_{\text {new }}\right)$ parameters presented on figure 1, with $\left|\Delta m_{\text {new }}^{2}\right|>1.5 \mathrm{eV}^{2}(95 \%$ C.L. $)$ and $\sin ^{2}\left(2 \Theta_{\text {new }}\right)=$ $0.14 \pm 0.18$ (95\% C.L.).

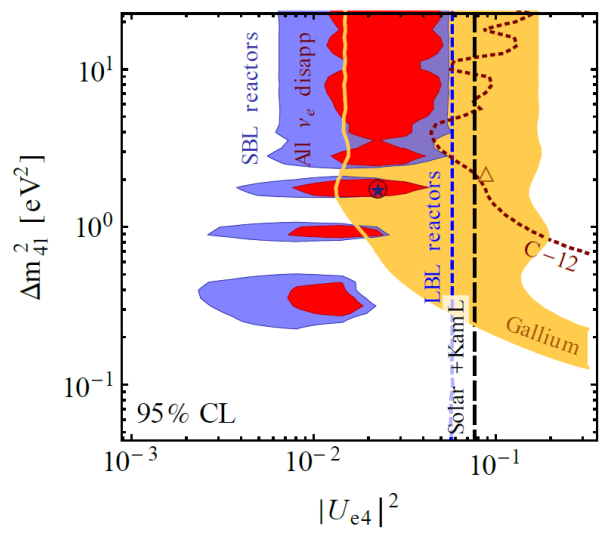

Figure 1: Allowed regions from the combination of $\mathrm{Ga}$ and RAA anomalies [5].

Concerning accelerator experiments, LSND results showed a total excess of $v_{e}$ events in a $v_{\mu}$ beam of 87.9 $\pm 22.4 \pm 6.0 v_{e}$-like events with 1993-1998 years of data. That suggested a $v$ flavor oscillations with a $\Delta m^{2} \in(0.2,10) \mathrm{eV}^{2} / c^{4}$. Since it was the very first time for this kind of observation, other projects came to cross-check LSND results. Recent MiniBooNE experiment verifies LSND excess and shows a consistent total $v_{e}+\bar{v}_{e}$ charged-current quasi-elastic excess of $460.5 \pm 95.8(4.8 \sigma)$ events [6]. However, the joint analysis of LSND and MiniBooNE data provided a controversial first best fit point at $\Delta m^{2}=0.041 \mathrm{eV}^{2}, \sin ^{2}(2 \Theta)=0.958$ [7] that motivated strong discussions into the neutrino community. In addition, MiniBooNE also showed that using other exotic MSW-Like Resonance Model could provide a better fit. Thus, $v_{e}$ anomalous appearance in accelerator experiments is still under study. Fermilab SBN project should adress these short baseline anomalies in the next 5 years [8]. 
Accelerator $v$ beams also perform studies looking at $v_{\mu}$ disappearance using near and far detectors, such as MINOS and MINOS+ experiments [9]. Results from $v_{\mu}$ disappearance are today in tension with LSND/MiniBooNE ones. MINOS provides an exclusion plot that rejects the main part of allowed region given by LSND and MiniBooNE, as well as a wide exclusion region with no evidence of $v_{\text {sterile }}$ in $\Delta m^{2}$ (from $\sim 10^{-2}$ up to $10^{3} \mathrm{eV}^{2}$ ) and $\sin ^{2}\left(\Theta_{24}\right)$ (from $\sim 10^{-2}$ to 1) [10].

The experiment/prediction discrepancy in neutrino fluxes is the question to be answered in the very near future, using short baseline reactor and accelerator $v$ experiments. The goal is to know if observed anomalies are due to the presence of the $4^{\text {th }} v_{\text {sterile }}$ flavor or due to errors into data analysis procedures. In the next sections this review is focused on reactor neutrinos experiments, both past and present ones.

\section{Light $v_{\text {sterile }}$ search with reactor neutrino experiments}

Initial main goal of reactor neutrino experiments was the measurement of $\Theta_{13}$ mixing angle. However, experiments such as Daya Bay, Double Chooz, RENO also provided useful information concerning $v_{\text {sterile }}$. Nowadays, the very short baseline reactor experiments, specially designed for $v_{\text {sterile }}$ searches, are in charge of explaining the RAA and other additional questions such as the spectral distorsion of reactor neutrino flux into 4-6 MeV range, also called as the "bump".

\subsection{Reactor $v$ experiments measuring $\Theta_{13}$}

These experiments use commercial reactor cores, placing near and far detectors at certain distances from nuclear power plants. Detector usually consists of liquid scintillator dopped with $G d$ and the $v$ is counted via the inverse beta decay reaction (IBD) $\bar{v}_{e}+p \rightarrow e^{+}+n$. The survival probability of $v_{e}$ can be noted as $P_{e e}(L)=1-\sin ^{2}\left(2 \Theta_{i}\right) \sin ^{2}\left(1.27 \frac{\Delta m_{i}^{2} L}{E_{\bar{v}_{e}}}\right)$, where $\Delta m_{i}^{2}$ is in $\mathrm{eV}^{2}, L$ in $\mathrm{m}, E_{\bar{v}_{e}}$ in MeV. This survival probability $P_{e e}(L)$ depends on the distance core-detector and neutrino energy.

Besides the $\Theta_{13}$ values, Daya Bay $[11,12]$, Double Chooz $[13,14]$ and RENO $[15,16]$ provided also ratios of measured/predicted reactor neutrino fluxes (see table 1) and exclusion of light $v_{\text {sterile }}$ in the very low region of $\left|\Delta m_{41}^{2}\right|<10^{-1} \mathrm{eV}^{2}$.

$\begin{array}{cc} & \text { Data/Prediction } \\ \text { Daya Bay (1230 days) } & 0.952 \pm 0.014 \text { (exp.) } \pm 0.023 \text { (model) } \\ \text { Double Chooz (101 days) } & 0.944 \pm 0.016 \text { (stat.) } \pm 0.040 \text { (syst.) } \\ \text { RENO (2200 days) Huber Mueller model } & 0.924 \pm 0.018 \\ \text { RENO (2200 days) ILL Vogel model } & 0.966 \pm 0.019\end{array}$

Table 1: Reactor neutrino flux ratios Data/Prediction from Daya Bay [12], Double Chooz [14] and RENO experiments [16].

It can be seen that RAA is clearly observed. In addition, a particular $v$ spectrum distorsion was observed by the three experiments into the 4-6 MeV region, figure 2 shows the Daya Bay example. 


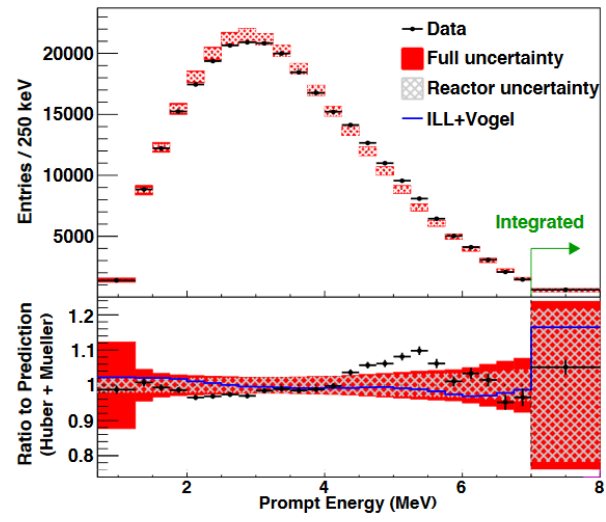

Figure 2: $v$ spectrum distorsion observed by Daya Bay into the 4-6 MeV region [12].

As it was mentioned before, commercial reactor cores were used in these experiments. This means that fuel composition analysis is complex since the initial core is already heterogeneous and the effect of fuel burn-up accentuates it even more. Thus, experiments using homogeneous core compositions, such as research compact reactors mainly formed by ${ }^{235} \mathrm{U}$, may provide better explanation of the observed $v$ spectrum distorsion.

Exclusion contours given by these experiments do not provide any evidence of sterile neutrino mixing for a very low $\left|\Delta m_{41}^{2}\right|<10^{-1} \mathrm{eV}^{2}$ region (see figure 3).
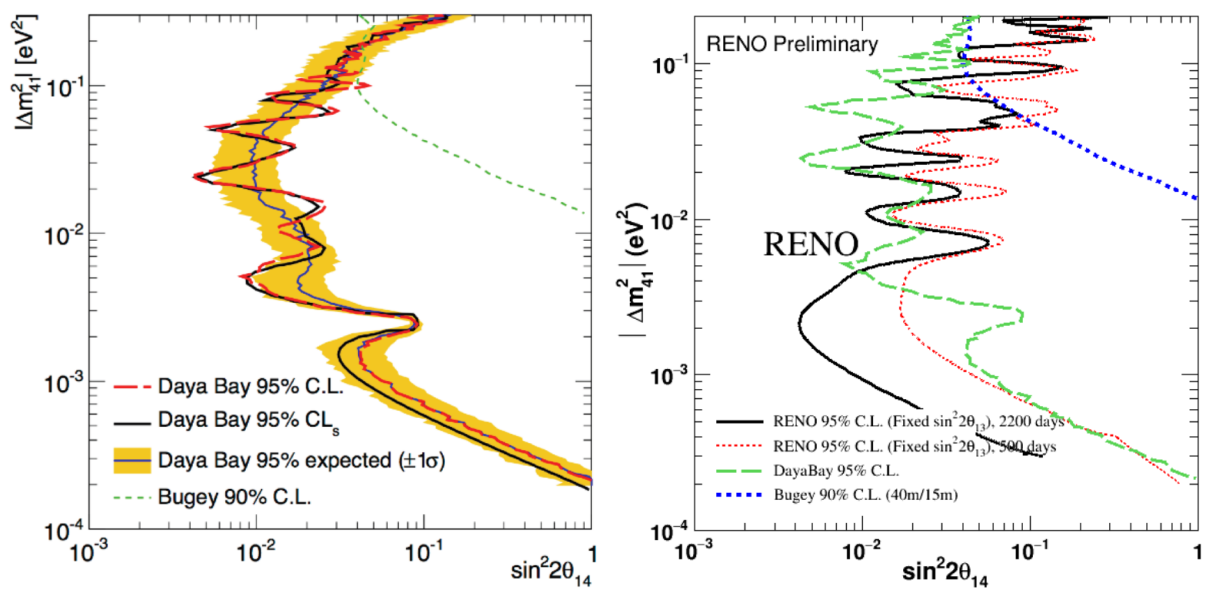

Figure 3: Exclusion contours for light $v_{\text {sterile }}$ by Daya Bay and RENO [11, 17].

In order to explore higher region of $\left|\Delta m_{41}^{2}\right|$ and verify the allowed region given by RAA+Ga combination from figure 1, very short baseline reactor $v$ experiments were designed and are currently taking data and providing results.

\subsection{Very short baseline reactor $v$ experiments searching for $v_{\text {sterile }}$}

Today's short baseline reactor experiments were specially designed for the $v_{\text {sterile }}$ searches $[18,19,20,22,23,24]$. However, they not always use similar detector design or reactor core. Tables 2, 3 present the experiments that are currently taking data. Core size and fuel composition impact on systematics and $v$ rates obtained by each experiment. 


$\begin{array}{cccccc} & \text { Core } \Phi & \text { Core } H & P_{t h} & { }^{235} \mathbf{U} \% & \text { Baseline (m) } \\ \text { NEOS } & 3.1 \mathrm{~m} & 3.8 \mathrm{~m} & 2.8 \mathrm{GW} & \sim 4 & 23.7 \\ \text { DANSS } & 3.2 \mathrm{~m} & 3.7 \mathrm{~m} & 3.1 \mathrm{GW} & \sim 4 & 10.7-12.7\end{array}$

Table 2: $v_{\text {sterile }}$ experiments using commercial low enriched ${ }^{235} \mathrm{U}$ reactors.

$\begin{array}{cccccc} & \text { Core } \Phi & \text { Core } H & P_{t h} & { }^{235} \mathbf{U} \% & \text { Baseline (m) } \\ \text { STEREO } & 40 \mathrm{~cm} & 80 \mathrm{~cm} & 58 \mathrm{MW} & 93 & 9-11 \\ \text { SOLID } & 50 \mathrm{~cm} & 90 \mathrm{~cm} & 50-80 \mathrm{MW} & 93 & 6-9 \\ \text { PROSPECT } & 44 \mathrm{~cm} & 51 \mathrm{~cm} & 85 \mathrm{MW} & >93 & 7-9 \\ \text { NEUTRINO-4 } & 42 \times 42 \mathrm{~cm} & 35 \mathrm{~cm} & 100 \mathrm{MW} & \sim 90 & 6-12\end{array}$

Table 3: $v_{\text {sterile }}$ experiments using compact highly enriched ${ }^{235} \mathrm{U}$ reactors.

All these projects revealed their first physics results concerning the IBD rates/day, ratios of $v$ fluxes and exclusion contours during 2018 (see table 4).

$\begin{array}{ccc} & \sigma / E \% & \text { average rate/day } \\ \text { NEOS } & 5 & \sim 1976 \\ \text { DANSS } & 17 & 4101 \pm 11 \\ \text { STEREO } & 9 & 396 \pm 4.7 \\ \text { SOLID(preliminary) } & 14 & \sim 1750 \text { ON \& } \sim 1375 \text { OFF } \\ \text { PROSPECT } & 4.5 & \sim 750 \\ \text { NEUTRINO-4 } & ? & \sim 200\end{array}$

Table 4: IBD average rates/day and energy resolution of ongoing short baseline reactor experiments.

In order to be independant from the $v$ spectrum distorsion into 4-6 MeV region, observed by Daya Bay, Double Chooz and RENO, all collaborations presented ratios of $v$ fluxes at different baselines.

Since only one baseline is available for NEOS, they provided $v$ reactor flux normalized to the Daya Bay spectrum. That gave place to discussions into the neutrino community concerning the coherence and consistency of choosing Daya Bay as the reference spectrum. Exclusion plot for $\left|\Delta m_{41}^{2}\right|>10^{-1} \mathrm{eV}^{2}$ region showed no strong evidence for light $v_{\text {sterile }}$ existence with $\Delta m^{2} \sim 1 \mathrm{eV}^{2}$, $\sin ^{2}(2 \Theta) \sim 0.1$ (see figure 4). Moreover, NEOS suggested the new best fit point at $\Delta m_{41}^{2}=1.73$ $\mathrm{eV}^{2}, \sin ^{2}\left(2 \Theta_{14}\right)=0.05$ (fig. 4). We should also keep in mind that $\mathrm{a} \sim 3 \mathrm{~m}$ large NEOS reactor core dimension might impact the amplitude of possible low energy $v$ oscillations. 

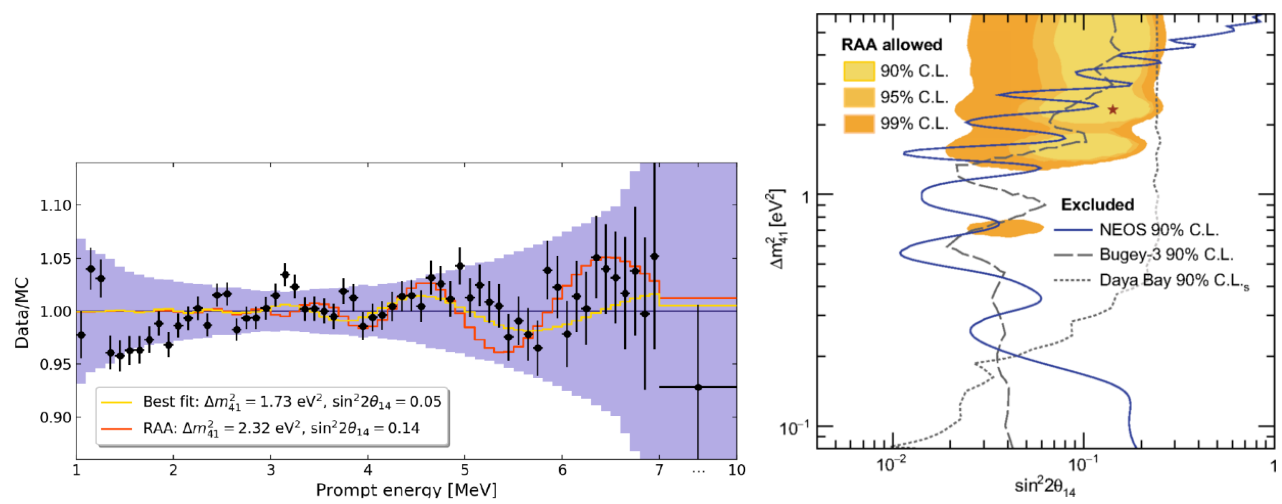

Figure 4: NEOS, $v$ reactor flux normalized to the Daya Bay spectrum and exclusion contours [18].

DANSS collaboration, that uses detector placed under reactor core, provided $v$ reactor flux ratios between 3 different baselines: down $-12.7 \mathrm{~m}$, middle $-11.7 \mathrm{~m}$, top $-10.7 \mathrm{~m}$. DANSS excludes a large fraction of Ga and RAA allowed regions as well as the best fit $\Delta m_{41}^{2}=2.3 \mathrm{eV}^{2}$, $\sin ^{2}\left(2 \Theta_{14}\right)=0.14$ at $5 \sigma$ level. Suggested best fit point at $\Delta m_{41}^{2}=1.4 \mathrm{eV}^{2}, \sin ^{2}\left(2 \Theta_{14}\right)=0.05$ with $2.8 \sigma$ significance still has to be studied taking into account systematic uncertainties after collection of more data. Finally, same as for NEOS, attention should be paid to a possible damping of low energy $v$ oscillations due to large core size.

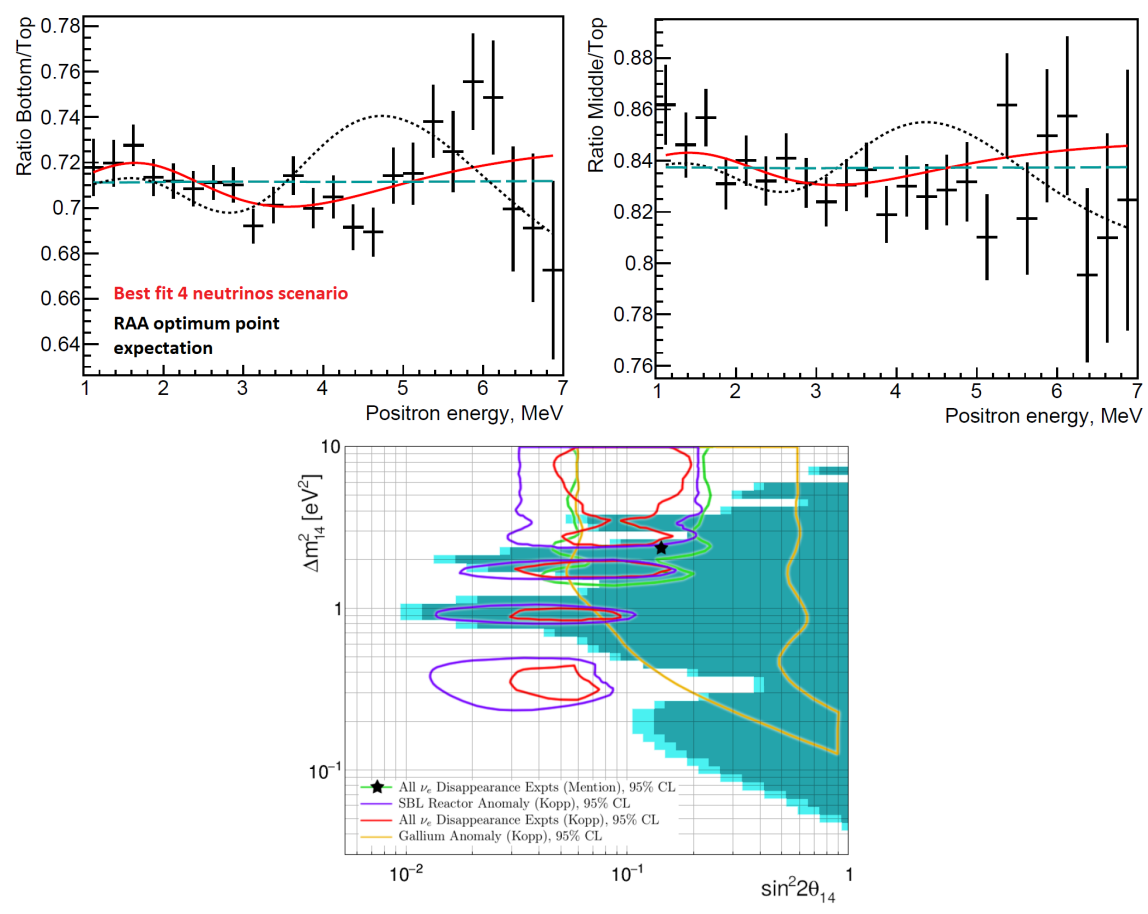

Figure 5: DANSS, $v$ reactor flux ratios at different baselines and exclusion contours [19].

STEREO collaboration, that uses a compact highly enriched ${ }^{235} \mathrm{U}$ core, presented $v$ flux ratios for 5 different baselines and exclusion contours (see figure 6). STEREO results are compatible with the null oscillation hypothesis and RAA best fit $\Delta m_{41}^{2}=2.4 \mathrm{eV}^{2}, \sin ^{2}\left(2 \Theta_{14}\right)=0.14$ is excluded 
at $98 \%$ C. L [21]. STEREO is an ongoing experiment that expects taking data until end of 2019 reaching $\geq 300$ reactor $\mathrm{ON}$ data days.

PROSPECT results give similar conclusions as the already mentioned experiments. Neutrino flux ratios (fig. 7) do not show any significant deviations from unity and are compatible with the no-oscillation hypothesis. Exclusion contours with 33 data days conclude with RAA best fit value excluded at $2.2 \sigma$ level.
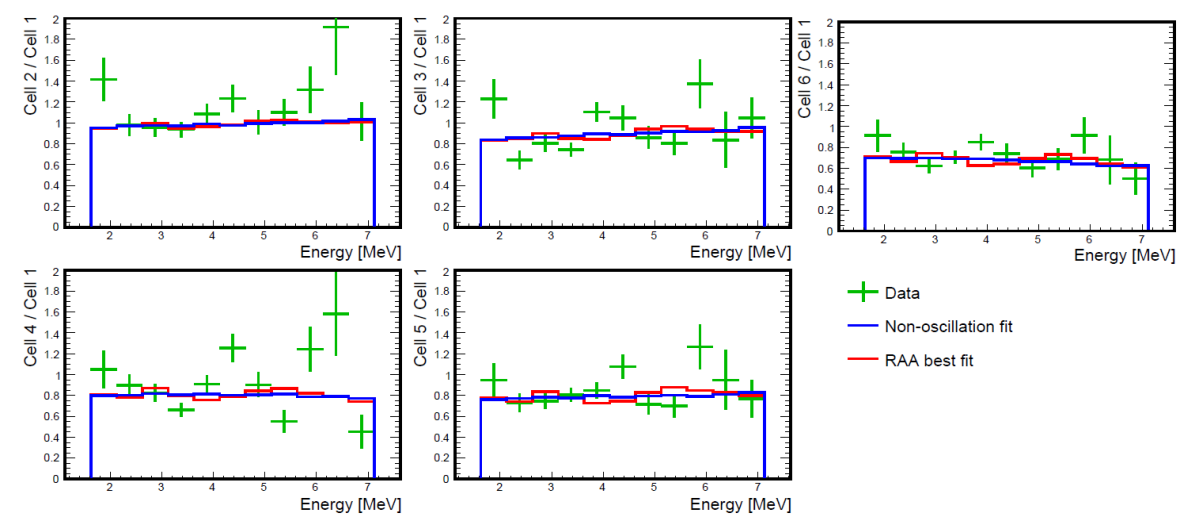

- RAA best fit

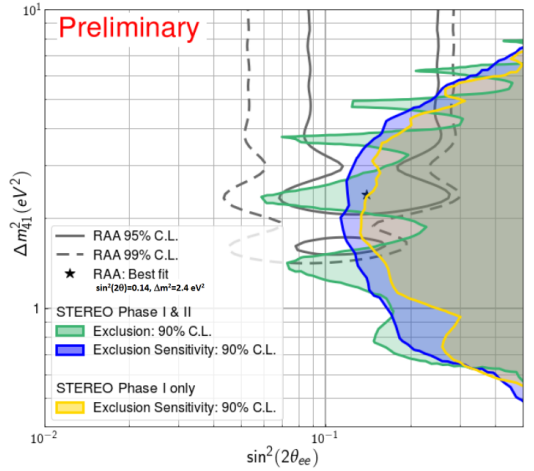

Figure 6: STEREO, $v$ reactor flux ratios at different baselines and exclusion contours (66+47 data days) [21].
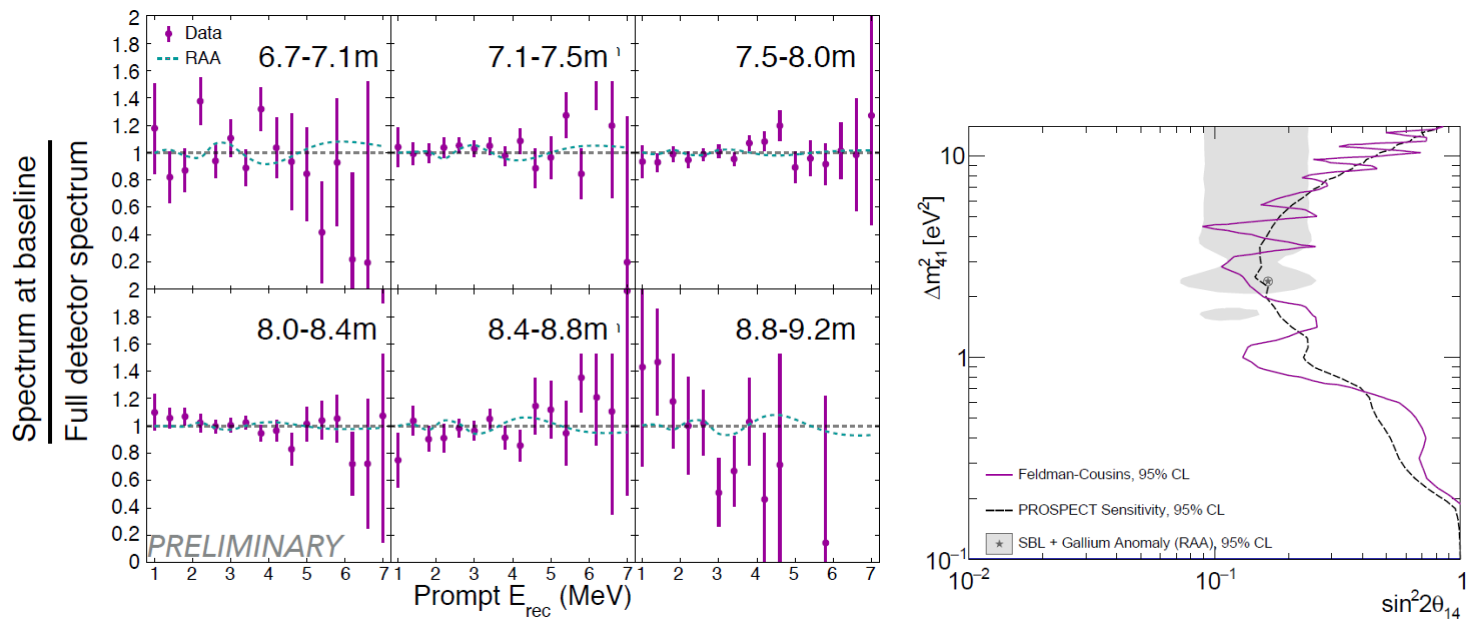

Figure 7: PROSPECT, $v$ reactor flux ratios at different baselines and exclusion contours (33 data days) [23]. 
Concerning SOLID, first physics results are under study. The expected sensitivity with 450 data days should cover a large part of RAA and Ga allowed regions.

Concerning the $v$ spectrum distorsion in 4-6 MeV region, more clear explanations may be given by measurements of the pure ${ }^{235} \mathrm{U}$ spectrum to be provided by STEREO, SOLID and PROSPECT in soon future.

Recently, NEUTRINO-4 collaboration communication [25], presented $v$ flux ratios claiming the first observation of $v_{\text {sterile }}$ oscillation. That obviously induced an hectic debate into the neutrino community which is today far away from being solved.

NEUTRINO-4 results were given for 480 days with the reactor ON and 278 days with the reactor OFF operation. NEUTRINO-4 observed neither instrumental systematic errors nor effects caused by structure of the detector in their analysis. However, cosmic background is difficult to master as well as inconsistency into energy spectrum when comparing measured and predicted $v$ spectra. Figure 8 shows the flux ratio and excluded/allowed regions provided by NEUTRINO-4.
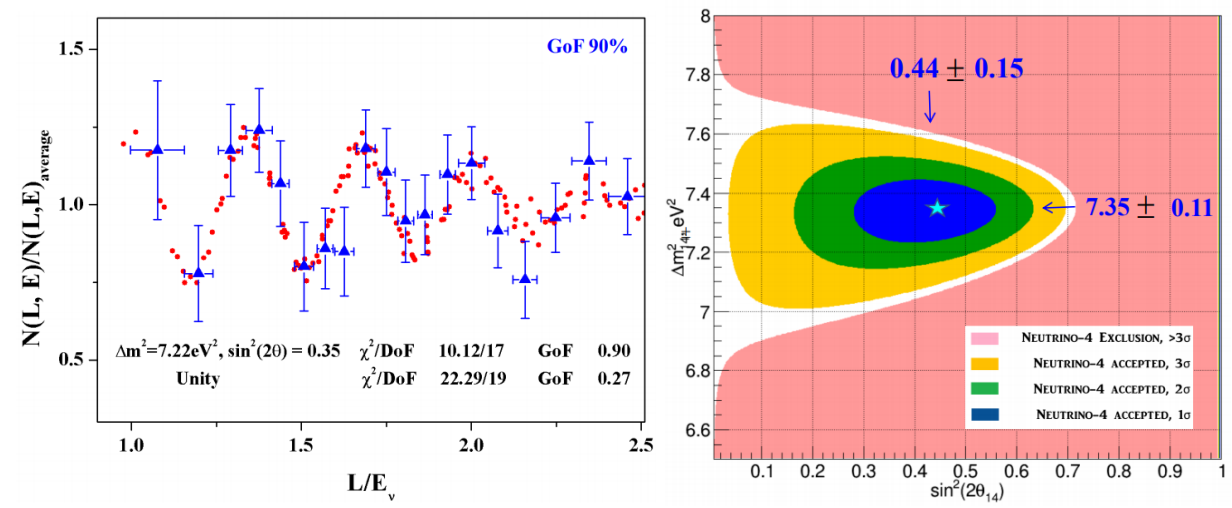

Figure 8: NEUTRINO-4, $v$ reactor flux ratios at different baselines and excluded/allowed regions (480 reactor $\mathrm{ON}$ data days) [24].

NEUTRINO-4 claims to observe the oscillation effect at C.L. $99.7 \%(3 \sigma)$ in vicinity of $\Delta m_{14}^{2}=7 \mathrm{eV}^{2}, \sin ^{2}\left(2 \Theta_{14}\right)=0.4$. However, today these parameters are not fully verified by other short baseline experiments using ${ }^{235} \mathrm{U}$ enriched cores. Results from PROSPECT, STEREO, with larger statistics might refute or confirm NEUTRINO-4 observation (see figure 9).

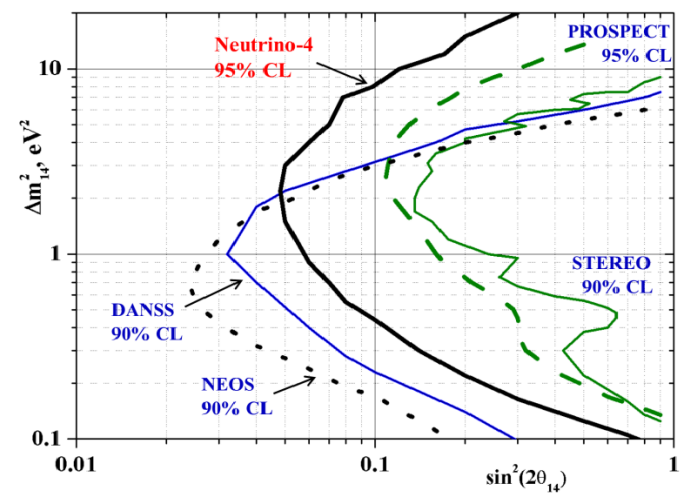

Figure 9: Sensitivity regions of short baseline reactor experiments compared to NEUTRINO-4 [25]. 
Generally, todays's available data statistics show no evidence of light $v_{\text {sterile }}$ mixing at short baselines and axcludes RAA best fit $\Delta m_{41}^{2}=2.4 \mathrm{eV}^{2}, \sin ^{2}\left(2 \Theta_{14}\right)=0.14$ at more than $90 \%$ C.L. Exception is made for NEUTRINO-4 that claims $v_{\text {sterile }}$ oscillation around $\Delta m_{14}^{2}=7 \mathrm{eV}^{2}, \sin ^{2}\left(2 \Theta_{14}\right)=$ 0.4. This last result has still to be accurately studied and compared to other experiments once more data days are available.

\section{Conclusion}

Observed $v$ anomalies such as LSND, Ga and RAA have motivated $v_{\text {sterile }}$ dedicated searches at very short reactor baselines and highly enriched ${ }^{235} \mathrm{U}$ cores. Ongoing projects NEOS and DANSS, using commercial reactors, excluded the RAA best fit $\Delta m_{41}^{2}=2.4 \mathrm{eV}^{2}, \sin ^{2}\left(2 \Theta_{14}\right)=$ 0.14 at more than $90 \%$ C.L. and their combined analysis [26, 27] suggested new best fit values $\Delta m_{41}^{2}=1.29 \pm 0.03 \mathrm{eV}^{2}, \sin ^{2}\left(2 \Theta_{e e}\right)=0.049 \pm 0.011$ (see figure 10).
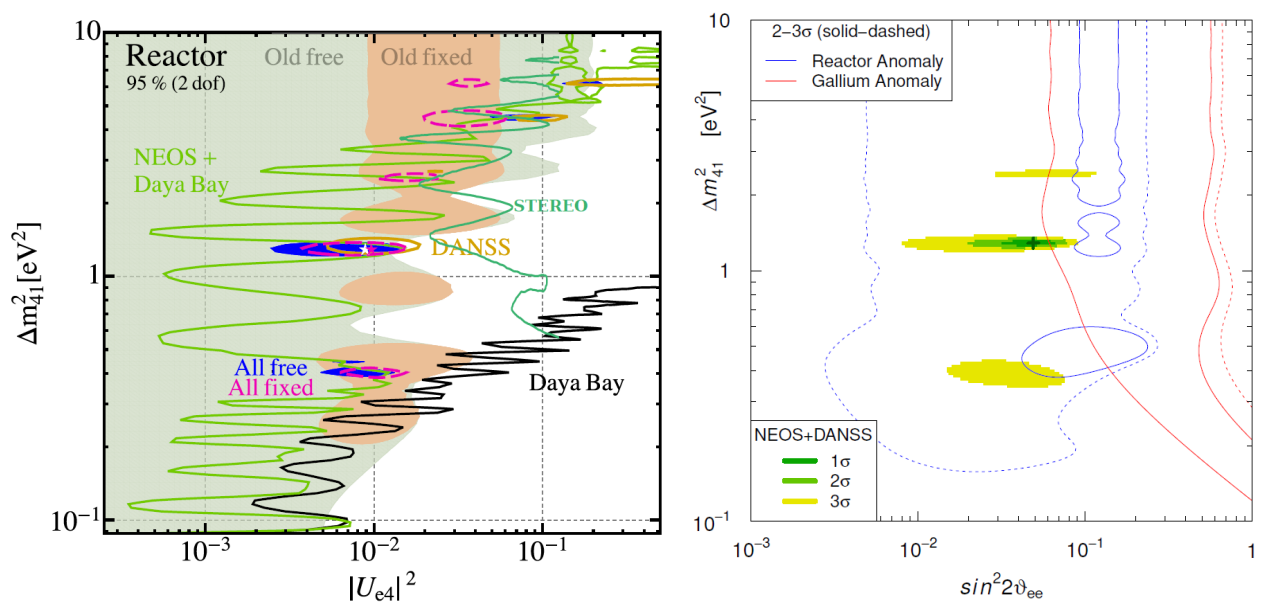

Figure 10: New best fit $\left(\Delta m_{41}^{2}, \sin ^{2}\left(2 \Theta_{14}\right)\right)$ favored by the combined NEOS+DANSS analyses [26, 27].

However, it should be kept in mind that more data are being expected and studies of systematics are under progress for some experiments (for example, DANSS). Thus, the conclusion about new best fit parameters is not an absolut statement at this stage.

Large part of RAA and Ga anomaly region was excluded by NEOS, DANSS, STEREO and PROSPECT projects. NEUTRINO-4 suggested oscillation observation for higher $\Delta m_{41}^{2}$ value which still has to be investigated and compared to other experiments (for example, PROSPECT).

Near future measurements of pure ${ }^{235} \mathrm{U} v$ spectrum by STEREO, PROSPECT and SOLID should bring more light on $v$ reactor spectrum distorsion into 4-6 MeV region.

Thus, future 2019 communications from reactor $v$ experiments will probably be the ones confirming or refuting the light $v_{\text {sterile }}$ hypothesis.

\section{References}

[1] J. N. Abdurashitov et al., GALLEX, "Measurement of the solar neutrino capture rate with gallium metal. III: Results for the 2002 - 2007 data-taking period.", Physical Review C, 80 (2009) 015807. 
[2] C. Giunti, M. Laveder, "Statistical Significance of the Gallium Anomaly", Physical Review C, 83 (2011) 065504.

[3] G. Mention et al., "The Reactor Antineutrino Anomaly", Physical Review D, 83 (2011) 073006.

[4] A. Aguilar et al., LSND, "Evidence for Neutrino Oscillations from the Observation of $\bar{v}_{e}$ Appearance in a $\bar{v}_{\mu}$ Beam", Physical Review D, 64 (2001) 112007.

[5] J. Kopp et al., "Sterile neutrino oscillations: the global picture", Journal of High Energy Physics, 50 (2013).

[6] A. A. Aguilar-Arevalo et al., MINIBOONE, "Observation of a Significant Excess of Electron-Like Events in the MiniBooNE Short-Baseline Neutrino Experiment", [arXiv:1805.12028v1 [hep-ex], 2018].

[7] En-Chuan Huang, "Updated MiniBooNE $v_{\mu} \rightarrow v_{e}$ Oscillation on Results", talk given at Neutrino2018 conference.

[8] R. Guenette, "MicroBooNE and the Future SBN Program", talk given at Neutrino2018 conference.

[9] P. Adamson et al., MINOS, "Search for sterile neutrinos in MINOS and MINOS+ using a two-detector fit", [arXiv:1710.06488, 2017].

[10] A. Aurisano, "Recent Results from MINOS and MINOS+", talk given at Neutrino2018 conference.

[11] F. P. An et al., Daya Bay, "Improved measurement of the reactor antineutrino flux and spectrum at Daya Bay", Chinese Physics C, 41 (2017) 013002.

[12] J. Pedro Ochoa-Ricoux, "Latest results from Daya Bay", talk given at Neutrino2018 conference.

[13] Y. Abe et al, Double Chooz, "Indication of reactor $v_{e}$ disappearance in the Double Chooz experiment", Physical Review Letters, 108 (2012) 131801.

[14] Ch. Buck, "New Results from the Double Chooz Experiment", talk given at Neutrino2018 conference.

[15] J.H. Choi et al., RENO, "Observation of energy and baseline dependent reactor antineutrino disappearance in the RENO experiment", Physical Review Letters, 116 (2015) 211801.

[16] H. Seo, "New Results from RENO", talk given at ICHEP2018 conference.

[17] Yu, Intae, "Recent Results from RENO", talk given at Neutrino2018 conference.

[18] Y. J. Ko et al., NEOS, "Sterile Neutrino Search at the NEOS Experiment", Physical Review Letters, 118 (2017) 121802.

[19] I. Alekseev et al., DANSS, "Search for sterile neutrinos at the DANSS experiment", Physics Letters B, 787 (2018) 56-63.

[20] H. Almazan et al., STEREO, "Sterile Neutrino Constraints from the STEREO Experiment with 66 Days of Reactor-On Data", Physical Review Letters, 121 (2018) 161801.

[21] J. Lamblin, "Latest results of the STEREO experiment", talk given at Neutrino2018 conference.

[22] G. N. Van Remortel, "SoLid: Search for Oscillations with Lithium-6 Detector at the SCK-CEN BR2 reactor", talk given at Neutrino2018 conference.

[23] PROSPECT collaboration, "First search for short-baseline neutrino oscillations at HFIR with PROSPECT", [arXiv:1806.027, 2018].

[24] A. P. Serebrov et al., NEUTRINO-4, "The first observation of effect of oscillation in Neutrino-4 experiment on search for sterile neutrino", [arXiv:1809.10561, 2018]. 
[25] A. P. Serebrov et al., "The first observation of effect of oscillation in Neutrino-4 experiment on search for sterile neutrino", talk given at Applied Antineutrino Physics 2018 Workshop, California, USA.

[26] M. Dentler et al., "Updated global analysis of neutrino oscillations in the presence of eV-scale sterile neutrinos", Journal of High Energy Physics, 10 (2018).

[27] S. Gariazzo et al., "Model-Independent v $v_{e}$ Short-Baseline Oscillations from Reactor Spectral Ratios", Physics Letters B, 782 (2018) 13-21. 